\title{
Improved mechanical properties of HDPE/nano-alumina composite through silane coupling agent
}

\begin{abstract}
The effect of silane coupling agent to mechanical properties of HDPE/nano-alumina composite was investigated. Nano-alumina used in this study was produced by using sol-gel method. The shape and size of nano-alumina were determined by using TEM. Prior to the usage of nano alumina as a filler in the polymer matrix, (3-methacryloxypropyl) trimethoxysilane (MPS) was used to functionalize the nanoparticles due to its bifunctional nature: hydrolysable group (i $\mathrm{Si}(\mathrm{OCH} 3) 3$ ) and unsaturated carbon-carbon double bond. The XRD result shows diffraction spectrum of the synthesized nano-alumina. In order to improve the mechanical properties of the composite, nanoalumina was treated with (3methacryloxypropyl) trimethoxysilane (MPS). A high tensile strength, is achieved at $4 \mathrm{wt} \%$ of MPS.
\end{abstract}

Keyword: Composite; Coupling agent; Polyethylene; Silane; Tensile strength 